\title{
Laboratory modelling of the transfer processes between the ocean and atmosphere in the boundary layers
}

\author{
Daniil Sergeev ${ }^{1,2^{*}}$, Alexander Kandaurov ${ }^{1,2}$, Yuliya Troitskaya ${ }^{1,2}$ and Maxim Vdovin ${ }^{1,2}$ \\ ${ }^{1}$ Institute of applied physics RAS, Geophysical Research Division, 603950 Ulyanova st. 46, Nizhny Novgorod, Russia \\ ${ }^{2}$ Lobachevsky state university of Nizhny Novgorod, 603950 Prospect Gagarina 23, Nizhny Novgorod, Russia
}

\begin{abstract}
The processes of momentum and heat transfer between ocean and atmosphere in the boundary layer were investigated within laboratory modeling for a wide range of wind speed and surface wave including hurricane conditions. Experiments were carried out on the Wind-Wave Flume of the Large Thermostratified Tank of IAP RAS. A special net located under the surface at different depths allows to vary parameters of surface waves independently on wind parameters. Theory of self-similarity of air flow parameters in the flume was used to calculate values aerodynamic and heat transfer coefficients from the measured velocity and temperature profiles by Pito and hotfilm gauges respectively. Simultaneous measurements of surface elevation with system wire allow to obtain spectra and integral parameters of waves. It was demonstrated that in contrast to the drag coefficient, heat transfer coefficient is virtually independent of wind speed and wave parameters to the moment of the beginning of spray generation and then increases rapidly.
\end{abstract}

\section{Introduction}

The main quantitative characteristics of atmosphere and ocean turbulent exchange are fluxes of momentum, heat and moisture. These fluxes play an important role in many aspects of meteorological and oceanographic research, including climate modeling, weather forecasting, modeling of boundary-layer processes etc. Turbulent exchange of energy and momentum between the ocean surfaces and the atmosphere to a large extent controls the energy and water cycle and general circulation of the ocean and the atmosphere. At the boundary-layer scale, the fluxes in question control generation of waves and development of the upper ocean mixed layer. In numerical weather prediction and climate modeling, the air-sea fluxes are parameterized through mean-flow meteorological parameters. Turbulent momentum $\left\langle u^{\prime} w^{\prime}\right\rangle=u_{*}^{2}$, heat $\left\langle T^{\prime} w^{\prime}\right\rangle$ and moisture $\left\langle q^{\prime} w^{\prime}\right\rangle$ fluxes are expressed via bulk formulas through the following meteorological parameters measured at a reference level (usually $h_{10}=10 \mathrm{~m}$ above the water surface): the $10 \mathrm{~m}$ wind speed $U_{10}$, the difference between the $10 \mathrm{~m}$ air and water temperature $\Delta T_{10}$ and the difference of relative moisture at the water level and 10 $\mathrm{m}$ above the water surface $\Delta q_{10}$ :

$$
\begin{aligned}
& \left\langle u^{\prime} w^{\prime}\right\rangle=C_{D} U_{10}^{2} \\
& \left\langle T^{\prime} w^{\prime}\right\rangle=C_{T} U_{10} \Delta T_{10} \\
& \left\langle q^{\prime} w^{\prime}\right\rangle=C_{E} U_{10} \Delta q_{10}
\end{aligned}
$$

The dimensionless exchange coefficients; drag coefficient $C_{D}$, the Stanton number $C_{T}$ and Dalton number $C_{E}$ are dependent both on wind speed and density stratification of the marine atmospheric boundary layer (MABL) and on the effective roughness of the water surface. The heat and moisture transfer coefficients are usually considered equal (see [1]), then here only the Stanton number and grad coefficient will be investigated.

According to Monin-Obukhov similarity theory [2] the vertical profiles of velocity and temperature in a stratified MABL are:

$$
\begin{gathered}
u(z)=\frac{u_{*}}{\kappa} \ln \frac{z}{z_{0}}+\Phi_{m}\left(\frac{z}{L}\right) ; \\
T(z)-T(0)=\frac{\left\langle T^{\prime} w^{\prime}\right\rangle}{\kappa u_{*}} \operatorname{Pr}_{t} \ln \frac{z}{z_{0 T}}+\Phi_{T}\left(\frac{z}{L}\right) .
\end{gathered}
$$

Here

$$
L=\frac{u_{*}^{3}}{\kappa \frac{g}{T}\left\langle T w^{\prime}\right\rangle}
$$

Monin-Obukhov length scale (see [2]), $\operatorname{Pr}_{t}$ - turbulent Prandtl number, $z_{0}$ и $z_{0 T}$ are roughness momentum and heat lengths. When $z<<L$ functions $\Phi_{m}(z / L)=\Phi_{T}(z / L)$ cease, then following from (2), (3) the velocity and temperature profiles $u(z)$ and $T(z)$ are logarithmic. 
It is easy to see that, comparing definitions for the exchange coefficients (1) with expressions for the velocity profile (2) and temperature profiles (3) gives that the $C_{D}$ and $C_{T}$ depend on the characteristics of the stratification of the MABL, defined by the Obukhov scale, and on the properties of the underlying surface parameterized by roughness height. A convenient expression for the latter are the coefficients of turbulent exchange at neutral stratification:

$$
\begin{gathered}
C_{D 10 N}=\frac{\kappa^{2}}{\left(\ln \left(h_{10} / z_{0}\right)\right)^{2}} \\
C_{T 10 N}=\frac{\kappa^{2}}{\operatorname{Pr}_{t} \ln \left(h_{10} / z_{0}\right) \ln \left(h_{10} / z_{0 T}\right)}=\frac{\kappa \sqrt{C_{D 10 N}}}{\operatorname{Pr}_{t}\left|\ln \left(\frac{h_{10}}{z_{0 T}}\right)\right|}
\end{gathered}
$$

where $h_{10}=10 \mathrm{~m}$.

Obviously, the equations (5) and (6) can be regarded as alternative definitions of roughness parameters $z_{0}$ and $z_{0 T}$

Parameterization of roughness parameters or the exchange coefficients used in the models of atmospheric circulation, are reduced to their dependencies on wind speed [3], and in the advanced models [4] on the wave age. The problem of dependencies of the exchange coefficients in MABL on wind speed is closely connected with the question of the influence of surface waves and spray, i.e. the consequence of wind forcing, on the exchange of the momentum and mass between the atmosphere and the ocean. The effect of waves on the momentum transfer has been studied by many authors (see, for example, [5-8] and references therein). It is shown that the momentum transfer by wave disturbances, or the form drag, increases with the increasing wind speed accompanied by the widening of the wave spectra. The decrease of the turbulent momentum flux near the water surface leads to the decrease of the wind speed at the standard height and increase of the drag coefficient and the surface roughness parameter.

The influence of wave disturbances on the heat transfer is weaker than on the momentum transfer, because even at moderate wind speeds the heat flux controlled by wave-induced disturbances is a fraction of percentage of the turbulent heat flux (see, discussion of this issue, for example, in [9]). In connection with it, the theoretical model $[10,11]$ does not regard the wave heat flux, considering that the heat transfer is determined only by turbulent transport. In this case, the calculated dependence $C_{T 10 N}$ on the wind speed is significantly affected by the model of turbulent exchange. At the same time, all of the above mentioned models predict the increasing dependence of $C_{T 10 N}\left(U_{10}\right)$.

The experimental data confirm weaker dependence of the heat transfer coefficient $C_{T 10 N}$ on wind speed compared to $C_{D 10 N}\left(U_{10}\right)$ (see, for example, the field [1] and laboratory [12] data), but the character of the dependence is different in different models. A number of studies (e.g. the algorithm COARE 3.0 [1], field data
[13] and laboratory data [12]) indicate weak growth of $C_{T 10 N}$ with increasing wind speed $U_{10}$. Also, a weak growing dependence of the enthalpy transfer coefficient on the wind speed was found in the recent laboratory experiment [14], which studied heat transfer at a strong wind. At the same time, in [15] the dependence of $C_{T 10 N}$ on the wind speed was not found.

Increasing wind speed causes an exponential increase in the amount of spray in the atmospheric boundary surface layer (see, for example, [16] and references therein). The effect of the impact of spray on the drag coefficient is ambiguous. A number of studies argue that spray cause reducing of the aerodynamic resistance of the water surface at hurricane conditions. For example, according $[17,18]$ the mechanism of reducing the aerodynamic drag caused by the fact that spray create in the atmosphere surface layer efficient stable stratification in density, reducing turbulent exchange. In [19] another mechanism of reducing the resistance, associated with the peculiarities of the spray momentum exchange with the air flow, is analyzed. On the contrary, as is estimated in [20], the exchange of spray momentum with turbulent flow leads to an increase in surface drag. The effect of spray on the heat transfer in the atmospheric surface layer is discussed in the theoretical work [11], where in the case of stable stratification is shown a strong growth of heat transfer coefficient at a wind speed of 15-20 m/s.

The purpose of this study is to investigate the influence of wind speed and the water surface disturbances (waves and spray) on the exchange of the momentum and heat in MABL. The difficulty of such a study is connected with the fact that the waves are excited by the wind, and it is impossible to distinguish the contribution to the momentum and heat transfer caused by wave disturbances, from the influence of the peculiarities of turbulent transport, determined by the speed of the wind. The main difference of this study from the previous experimental studies and field observations is in the use of artificial, independent of wind speed, control of parameters of waves at a certain wind speed. This helped to obtain dependencies of the transfer coefficients on the wind speeds, parameters of waves and spray. It should be mentioned that flux transfer coefficient and heat transfer coefficient were measured simultaneously. It allowed to define scale of the temperature roughness $z_{0 T}$ characterizing heat transfer and independent on the flux transfer, as well as scale of surface wave roughness $z_{0}$. So it follows from (6) that the increase of $C_{T 10 N}$ is possible due to the growth of both $C_{D 10 N}$ and the temperature roughness $z_{0 T}$, which is determined by the properties of the underlying surface, quantifying the characteristics of waves and spray. Otherwise simultaneous measurment of $C_{D 10 N}$ and $C_{T 10 N}$ allowed to estimate its ratio, which an important parameter of hurricane development is the ratio of heat and momentum exchange coefficients, which determines the condition for maintaining a hurricane formulated by [21] as $C_{T} / C_{D}>0.75$.

According to the experiments [15], the ratio is close to 0.75 , but uncertainties of the field experiments are too large for definite conclusions. 
The paper has following structure. In the Section 2 experimental setup and a scheme of the adjustment of the surface roughness independently on the wind force are described. Section 3 is devoted to description of the measuring of the transfer coefficients by profiling method. The method of retrieving 3D spectra of the waves is described in the section 4 . In the sections 5 and 6 dependencies of the transfer coefficients on the parameters of wind flow, waves and influence of the sprays are discussed. Conclusion is in the section 7 .

\section{The description of the experimental setup for the study of heat and momentum exchange at strong winds}

The experiments were carried out on the Wind-Wave Flume of the Large Thermostratified Tank of IAP RAS (the overview see on Fig.1). The airflow channel with 10 $\mathrm{m}$ length has the section of $0,4 \times 0,4 \mathrm{~m}$ above the water surface. The airflow is created by a centrifugal fan, the frequency or rotation $f$ ranges from 7.5 to $50 \mathrm{~Hz}$. The airflow velocity at the axis of the channel is proportional to $f$ and varies from $4 \mathrm{~m} / \mathrm{s}$ to $20 \mathrm{~m} / \mathrm{s}$ (see Fig 3), which corresponds to the equivalent speed of $7-42 \mathrm{~m} / \mathrm{s}$. The detailed description of the experimental facility, the principles of creating and controlling the airflow is given in [22]. The general scheme of the experiments is shown in Fig. 2.

To create the temperature stratification of the surface layer of the wind, the air entering the channel, was heated to 35-40 $\mathrm{C}^{\mathrm{o}}$ (depending on the air flow speed).

The temperature on the water surface for all the experiments was maintained constant of about $15 \mathrm{C}^{\circ}$.

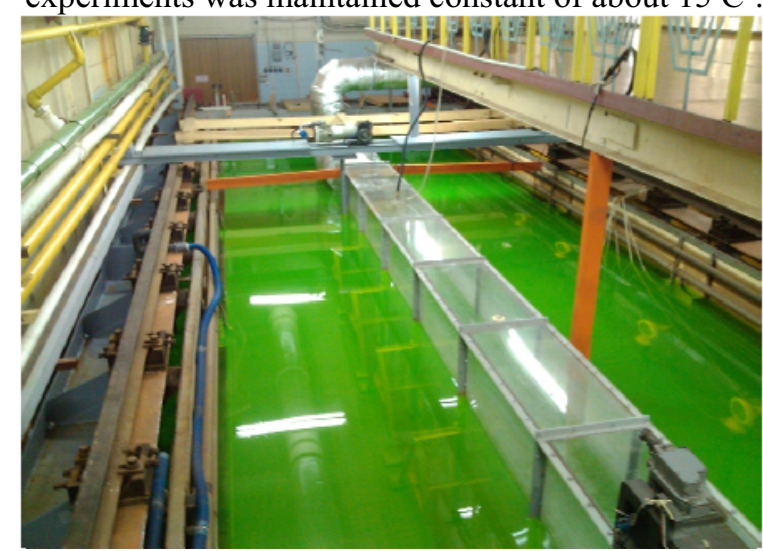

Fig. 1. Wind-Wave Flume of the Large Thermostratified Tank of IAP RAS (air channel in the center).

A special feature of this experiment is the ability to control surface waves irrespectively of the speed of the wind flow in the channel. For this purpose, the plastic net (mesh) $0.25 \mathrm{~mm}$ thick with a cell of $1.6 \times 1.6 \mathrm{~mm}$ has been stretched along the entire channel (first time was used in [23]). The net did not affect the heat exchange, but the characteristics of surface waves varied depending on its depth: the waves were absent when the net was located at the level of the undisturbed surface of the water, but at maximum depth $(33 \mathrm{~cm})$, it had practically no effect on the parameters of the surface disturbances for all wind speeds implemented on the facility.

Velocity and temperature profiles in the working section of the flume (at a distance of $6.5 \mathrm{~m}$ from the entrance channel) were measured simultaneously with the help of respectively the Pitot tube and hot film gauge, mounted on the vertical scanner. The L-shaped Pitot tube with the differential pressure transducer Baratron MKS 226 A provided the accuracy of velocity measurement $3 \mathrm{~cm} \mathrm{~s}^{-1}$. The accuracy of temperature measurements with hot film is $0.1{ }^{\circ} \mathrm{C}$.

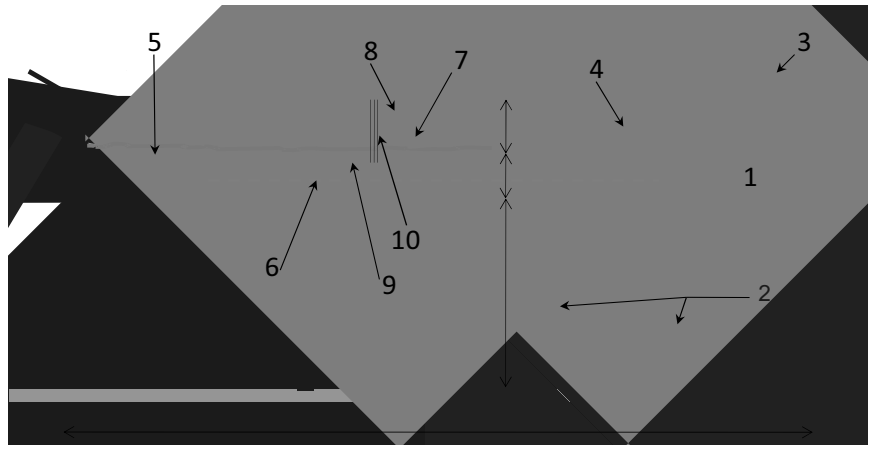

Fig. 2. General scheme of the experiments in Wind -Wave Flume. The sizes noted in $\mathrm{cm}$. (1)Wind-wave channel body, (2) vertical bearings , (3) convergent - diffusion section with a honeycomb, (4) hot film anemometer at the entrance, (5) wave absorber, (6) a net along the channel installed on the different depths (7) Pitot tube on a scanning system, (8) hot film thermometer on the same scanning, (9) a gauge for water temperature measurements. (10) three channel wire wavegauge.

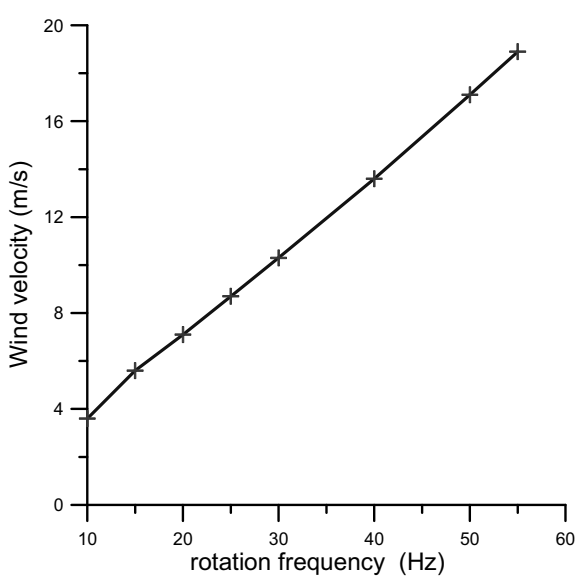

Fig. 3. Flow rate dependence on the fan rotation frequency

The scanning method with the consecutive height increment of $3-5 \mathrm{~mm}$ and accruing time of 1 minutes at each point was used. For each fixed wind parameters 2 profiles of velocity and temperature were measured for subsequent averaging. The lower level of scanning was located at a distance of $1 \mathrm{~cm}$ from the crests of the waves and depended on the wind speed, while the upper layer was $38 \mathrm{~cm}$ (in $2 \mathrm{~cm}$ below the upper lid of the channel). The temperature and wind speed at the inlet of the flume were controlled with the additional hot film gauge. Also, the temperature gauge was placed under water in the working section to measure the temperature of the surface layer of water. 


\section{The determination of heat and momentum flows on the velocity profiles of the air flow and temperature measured in the channel}

To determine the parameters of the atmospheric boundary layer the algorithm suggested in [22] was used, which generalizes the model of the boundary layer near a flat surface [24]. It is well known that the boundary layer on a smooth flat plate is composed of the viscous sublayer having a thickness of about $\delta_{v i s}=v_{a} / u_{*}$ (less than $1 \mathrm{~mm})$, positioned above the layer of constant fluxes having a thickness of about $0,15 \delta$, where $\delta$ is the boundary layer displacement thickness; and "the wake" part of the turbulent boundary layer, located higher up to the border of the displacement layer. In the turbulent boundary layer over a waved surface, an additional layer is generated, in which there is a transfer of momentum from the airflow to the wave perturbations on the water surface and the sum of turbulent and wave momentum fluxes is constant:

$$
\tau_{\text {turb }}(\eta)+\tau_{\text {wave }}(\eta)=u_{*}^{2} .
$$

and here $\tau_{\text {wave }}(\eta)$ decreases with increasing distance from the boundary on the scale $h_{\text {wave }}$, which, according to [25] can be determined from the following relationship

$$
k h_{\text {wave }}\left(\frac{\kappa V_{p}}{u_{*}}+\ln k z_{0}-\ln h_{\text {wave }} k\right)=\kappa^{2}
$$

Here $V_{p}$ and $k$ are the phase velocity and the wave number corresponding to the frequency of the peak of elevation surface spectrum. The numerical values of the parameters of wind and waves in the flume obtained below, give an estimate of $k h_{\text {wave }} \sim 0.01$. Here the condition $h_{\text {wave }}<0,15 \delta$ is observed, and within the layer of the constant momentum flux there is a layer of constant turbulent momentum flux $\tau_{\text {turb }}(\eta)=u_{*}^{2}$, in which the velocity depends on the vertical coordinate logarithmically.

As a rule, in wind tunnels and wind and wave channels, the thickness of the layer of constant fluxes is very small, for example, in the flume it was about $3 \mathrm{~cm}$. The measurement of the wind speed and temperature at such a small distance from the surface of waved water surface especially at strong winds is a difficult problem, mainly due to the effect of spray torn off the wave crests. However, the parameters of the layer of constant fluxes can be obtained from the measurements in the "wake" part of the turbulent boundary layer, as the profiles of velocity defect in the near-wall turbulent flows are selfsimilar:

$$
\frac{U_{\max }-U(z)}{u_{*}}=F\left(\frac{z}{\delta}\right),
$$

where $U_{\max }$ is the maximum speed in a turbulent boundary layer. According to [24] for non-gradient turbulent boundary layer at a flat plate or in the tube, the following approximation of the self-similar velocity profile

$$
U_{\max }-U(z)=\left\{\begin{array}{l}
u_{*}\left(-\frac{1}{\kappa} \ln (z / \delta)+\alpha\right) ; z / \delta<0.15, \\
\beta u_{*}(1-z / \delta)^{2} ; z / \delta>0.15
\end{array}\right.
$$

where $\kappa=0.4$ is the van Karman constant.

The constants $\alpha$ and $\beta$ can be obtained from the best fitting of the experimental data. In [24], the values of the constants $(\alpha=1, \quad \beta=9.6)$ for no-gradient turbulent boundary layer and $(\alpha=1, \beta=7.1)$ for the turbulent Poiseuille flow in a pipe. The experiments in the wind wave flume show that the profile of the velocity defect in the air flow above the waved water surface is also selfsimilar, and the velocity profile can be approximated by the expression (10). Determining the constants $\alpha$ and $\beta$ included the following data processing. The profiles of the flow velocity defect measured at a certain frequency of rotation of the fan and different positions of the net controlling waves, were expressed in terms of the selfsimilar coordinate $y=z / \delta$ and normalized by the curvature of the velocity profile $\beta u *$. Thus obtained dimensionless velocity defect profiles are shown in Fig. 4 for a number of fan rotation frequencies. It can be seen

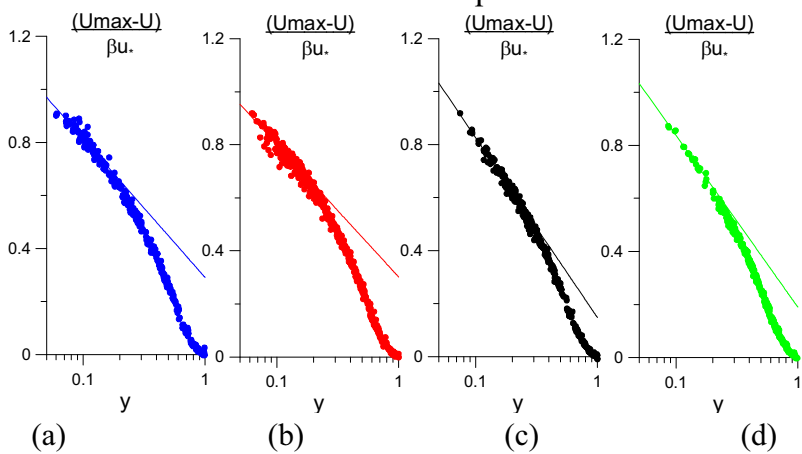

Fig. 4. Dimensionless velocity defect profiles for the following values of the fan speed: (a) $-20 \mathrm{~Hz}$, (b) - 30, (c)- $35 \mathrm{~Hz}$, (d) $40 \mathrm{~Hz}$.

that the experimental points collapse on certain curves, confirming the self-similarity of the profile of the flow velocity defect in the channel above the water surface. With this normalization the logarithmic part of the selfsimilar profile of the velocity defect has the form

$$
\frac{U_{\max }-U(z)}{\beta u_{*}}=\frac{1}{\beta}\left(-\frac{1}{\kappa} \ln (y)+\alpha\right)
$$

The parameters of the logarithmic boundary layer can be obtained from the measurements in the wake part of the turbulent boundary layer, first, retrieving the parameters of turbulent boundary layer $\left(U_{\max }\right.$ and $\delta$ ) from best fit of the experimental data by equation (10) for $z / \delta>0.15$ and then calculating the parameters of the logarithmic boundary layer by the following expressions:

$$
U(z)=\frac{u_{*}}{\kappa} \ln \left(z / z_{0}\right)
$$

where 


$$
z_{0}=\delta \exp \left(-\kappa U_{\max } / u_{*}+\alpha \kappa\right)
$$

Using $(12,13)$ it is possible to determine the wind speed at the standard height $H_{10}=10 \mathrm{~m}$ and calculate the drag coefficient

$$
C_{D 10 N}=\frac{\kappa^{2}}{\left(\kappa U_{\max } / u_{*}-\alpha \kappa+\ln \left(H_{10} / \delta\right)\right)^{2}}
$$

Similar processing carried out with the temperature profiles allows determining the Stanton number and temperature roughness from the temperature profiles in the channel. We used in this case the self-similarity of the profile of "the temperature defect", which was defined similarly to the expression (11) for the velocity defect:

$$
\frac{T_{\max }-T(z)}{T_{*}}=G\left(\frac{z}{\delta_{T}}\right)
$$

where $T_{*}=\frac{\left\langle T^{\prime} w^{\prime}\right\rangle}{u_{*}}$.

For the approximation of the self-similar dependence for the profile of "the temperature defect" we used the expression similar to (10):

$$
T_{\max }-T(z)=\left\{\begin{array}{l}
T_{*}\left(-\frac{1}{\kappa} \operatorname{Pr}_{t} \ln \left(z / \delta_{T}\right)+\alpha_{T}\right) \\
z / \delta_{T}<0.15 \\
\beta_{T} T_{*}\left(1-z / \delta_{T}\right)^{2} \\
z / \delta_{T}>0.15
\end{array}\right.
$$

For implementation the profiling method for measuring turbulent heat flux in MABL we need the turbulent Prandtl number $P r_{t}$. According to [3] $P r_{t}$ was assumed to be 0.85 . This value was also confirmed by direct numerical simulation of turbulent boundary layer above waved water surface [26]. To determine the constants $\alpha_{\mathrm{T}}$ and $\beta_{T}$ data processing was carried out as described above for the velocity profile. Similarly, the profiles of "the temperature defect" of the air flow were expressed in terms of the self-similar coordinate $y=z / \delta_{T}$ and normalized by the curvature of the temperature profile $\beta_{T} T_{*}$. Thus obtained dimensionless profiles of "the temperature defect" are shown in Fig. 5 for various fan rotation frequencies.

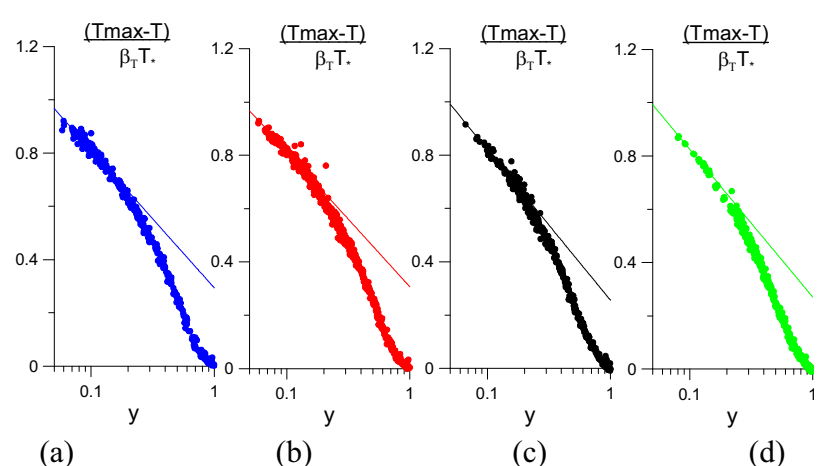

Fig. 5. Dimensionless profiles of "the temperature defect" for the following values of the fan speed: (a) - $20 \mathrm{~Hz}$, (b) - 30, (c)$35 \mathrm{~Hz}$, (d) - $40 \mathrm{~Hz}$.
The turbulent heat flux and temperature roughness were calculated by the measurements of the temperature in the "wake" part similar to the parameters of the logarithmic boundary layer (friction velocity and roughness parameter). The profile of the temperature defect was fitted by a polynomial of order 2 , which determines 3 parameters $\beta_{T} T_{*}, T_{\max }$ and $\delta_{T}$.

Knowing the constant $\beta_{T}$, taking into account the values of the van Karman constant and the Prandtl turbulent number, it is possible to find $T_{*}$, and taking the friction velocity found above, to determine the heat flux $\left\langle T^{\prime} w^{\prime}\right\rangle=T_{*} u_{*}$. Then the parameter of temperature roughness $z_{0 T}=\delta_{T} \exp \left(-\frac{\kappa}{\operatorname{Pr}_{t}} \frac{\left(T_{\max }-T_{w}\right)}{T_{*}}+\alpha_{T} \kappa\right)$ follows from (16), where $T_{w}$ - water surface temperature and the temperature profile in the layer of constant fluxes takes the form:

$$
T(z)=T_{w}+\frac{T_{*}}{\kappa} \operatorname{Pr}_{t} \ln \left(\frac{z}{z_{0 T}}\right)
$$

Using equation (17) for calculating the temperature difference between the surface water and air at a standard height $H_{10}=10 \mathrm{M}$ and equation (12) for $U_{10}$ gives then the Stanton number:

$$
\begin{aligned}
& C_{T 10 N}=\kappa^{2} /\left\{\left(\frac{\kappa U_{\max }}{u_{*}}-\alpha \kappa+\ln \left(\frac{H_{10}}{\delta}\right)\right) \times\right. \\
& \left.\times\left(\kappa \frac{\left(T_{\max }-T_{w}\right)}{\operatorname{Pr}_{t} T_{*}}-\alpha_{T} \kappa+\ln \left(\frac{H_{10}}{\delta}\right)\right)\right\}
\end{aligned}
$$

It follows directly from (17), that:

$$
\frac{T_{*}}{T\left(H_{10}\right)-T_{w}}=\frac{\kappa}{\operatorname{Pr}_{t} \ln \left(\frac{H_{10}}{z_{0 T}}\right)}
$$

depends only on temperature roughness. We will use the dimensionless parameter (19) instead of the dimensional temperature roughness for convenience of comparing with the available experimental data.

Comparing (14), (18) and (19) easy gives

$$
C_{T 10 N}=\frac{T_{*}}{T\left(H_{10}\right)-T_{w}} \sqrt{C_{D 10 N}}
$$

The expression (20) is useful for the analysis of dependences of the Stanton number, the parameter of temperature roughness and the coefficient of resistance of the wind and waves.

\section{Measuring parameters of surface waves}

The wind wave field parameters in the flume were measured by three wire gauges positioned in the corners of an equal-side triangle with $2.5 \mathrm{~cm}$ side, the data sampling rate was $100 \mathrm{~Hz}$. Three-dimensional spacetime spectra were obtained from the measured data by the algorithm FDM (Fourier Directional Method) [22], 
similar to Wavelet Directional Method (WDM), suggested in [27].

Then, the three-dimensional space-time spectrum $S(\omega, k, \theta)$ can be obtained similar to [26] by using the conditional averaging of the spectral amplitudes by $k$ and $\theta$.

Integrating the spectra obtained $S(\omega, k, \theta)$ by the wave number or frequency, gives temporary $S(\omega, \theta)$ or spatial $S(k, \theta)$ angular spectra, respectively. Integrating by $\theta$ gives the omnidirectional frequency and wave number spectra. The upper limit of the wave number spectrum is prescribed by the distance between the wave staffs $d, k_{u}=\pi / d$, in the used configuration $k_{u}=1,25 \mathrm{~cm}^{-1}$.

The advantage of FDM against WDM is due to its higher spectral resolution (see [22]). Judging by the balance of the frequency resolution and signal accumulation time later was selected the window size of 512 samples (5,12 seconds).

To quantify the wave field we used below the dimensionless mean square slope (m.s.s.) defined as the integral of the omnidirectional slope spectrum over the range of measured wave numbers, as it was determined in the [28]:

$$
\text { m.s.s. }=\int_{k_{0}}^{k_{u}} k^{2} S(k, \theta) k d k d \theta
$$

where $k_{0}$ is a wave number below the spectral peak.

The dependences of the wind speed, significant wave height and spray marker on the control parameters of the experiment (the fan rotation frequency and control net depth) are shown in Fig. 6. The figures show that the fan rotation frequency determines mainly the wind speed (Fig. 6a), the latter relatively weakly depends on the depth of the net (Fig. 6c), which on contrary strongly affects the wave height (see Fig. $6 b$ and d). Low dependency of wind speed on the net position can be explained by the low water surface drag under any wave conditions.

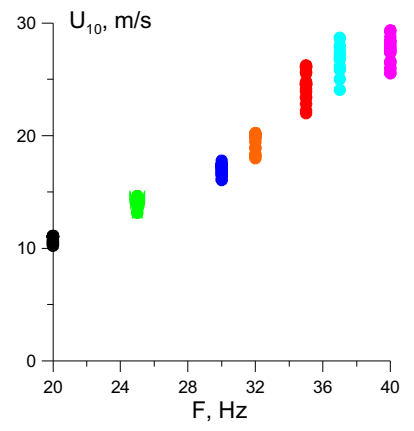

(a)

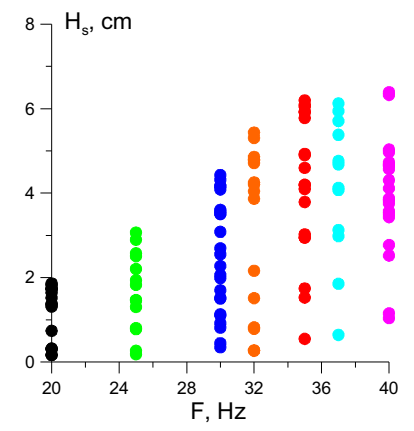

(b)

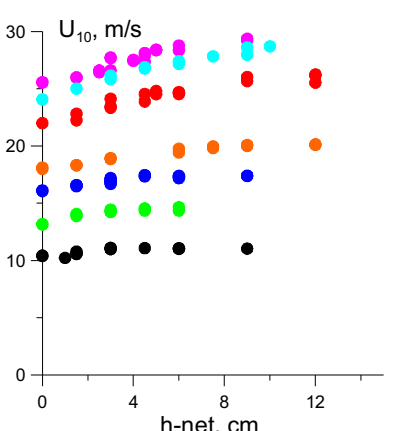

(c)

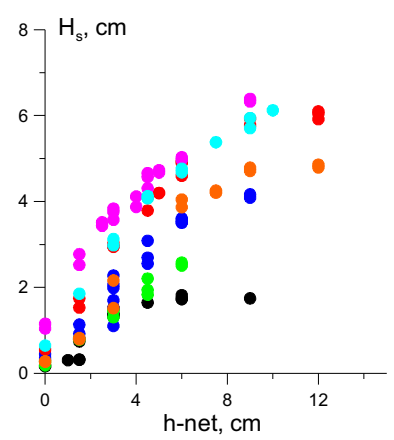

(d)
Fig.6. (a) equivalent wind speed $U_{10}$ on the $F$ (fan rotation speed), (b) significant wave height $H_{\mathrm{s}}$ on the $F$, (c) equivalent wind speed $U_{10}$ on the $h_{\text {net }}$ (depth of the net), (d) significant wave height $H_{\mathrm{s}}$ on the $h_{\text {net }}$. Black symbols corresponds fan speed $20 \mathrm{~Hz}$, green - $25 \mathrm{~Hz}$, blue $-30 \mathrm{~Hz}$, orange $-32 \mathrm{~Hz}$, red $-35 \mathrm{~Hz}$, sky - $37 \mathrm{~Hz}$, violet $-40 \mathrm{~Hz}$.

\section{Experimental dependencies of the turbulent exchange coefficients on wind velocity}

The obtained dependencies of exchange coefficients on parameters of wind are discussed in this section and compared with available field [29, 31-35] and laboratory $[14,30]$ experiments. Fig. 7 shows the dependency of drag coefficient on $10 \mathrm{~m}$ wind speed. It is obvious that, alternatively to the previous data, the noticeable spreading of the values of $C_{D 10 N}$ is observed at a certain frequency of fan rotation, which mainly prescribes the air-flow velocity (see Fig. 7a).

A similar strong spreading of the experimental points is seen in the dependency of the Stanton number on wind speed in Fig. 7b. The comparison with Fig. 7c shows, that the experimental spreading of the temperature roughness is significantly lower than for the Stanton number. It indicates the fact that the Stanton number, which is the product of $\sqrt{C_{D 10 N}}$ and the logarithm of the temperature roughness (see eq. 20), depends on the wave regime mostly through the dependence on surface roughness of $C_{D 10 N}$.

It is interesting to note that at $U_{10}>25 \mathrm{~m} / \mathrm{s}$ the Stanton number and the temperature roughness increases sharply, as can be seen below,due to the presence of spray. As a result, the ratio $C_{T 10 N} / C_{D 10 N}$ exceeds the marginal value 0.75 for all wind speeds (see Fig. $7 d$ ) confirming the Emanuel criterion of the hurricane maintaining.

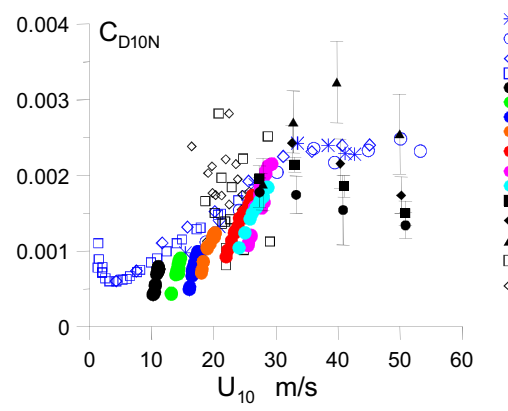

Donelan et.al. 2004

our data

Powel et.al., 2003

French et.al., 2007 Fabian French et.al., 2007 Isabel 
(a)

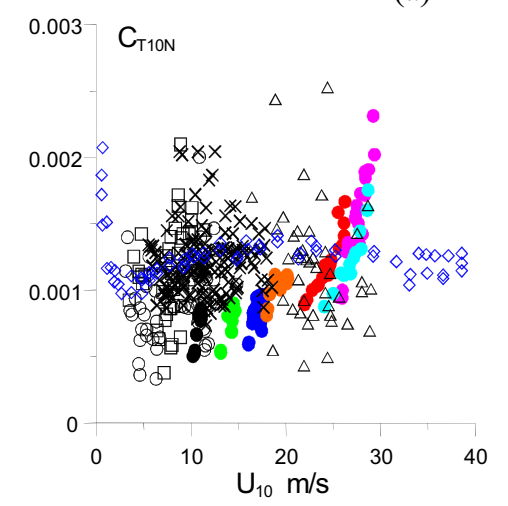

(b)

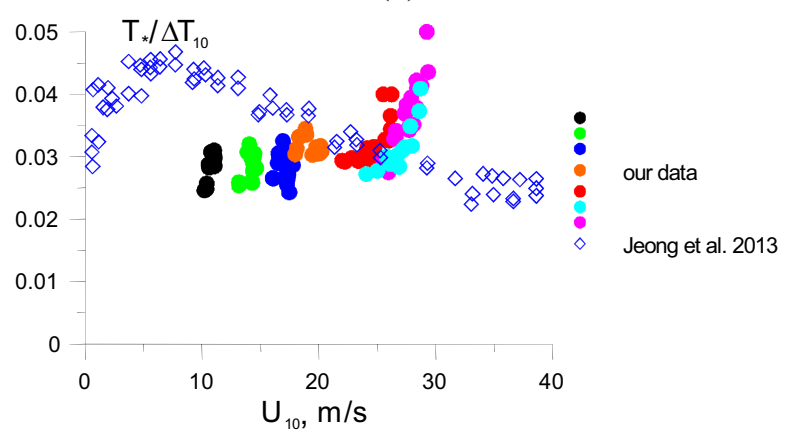

(c)

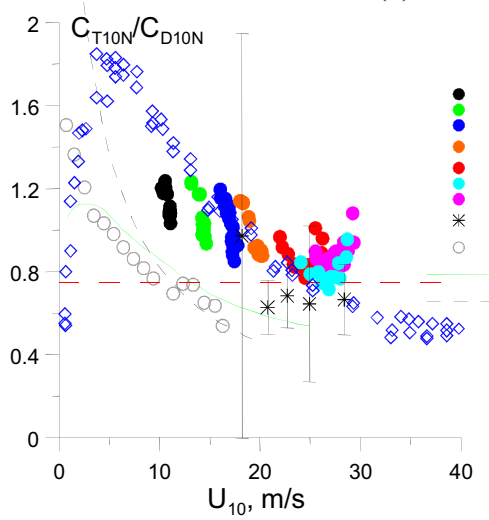

(d)

Fig.7. Dependencies: (a) of drag coefficient $C_{D 10 N}$ on $U_{10}$, (b) heat transfer coefficient $C_{\mathrm{T} 10 N}$ on $U_{10}$, (c) parameter of the temperature roughness on $U_{10}$. (d) the ratio $C_{T 10 N} / C_{D 10 N}$ on $U_{10}$. Red dashed line $C_{T 10 N} / C_{D 10 N}=0.75$. Colors closed circles corresponds results of this study (the same fan speed as on Fig. 6).

\section{The dependency of exchange coefficients on the parameters waves and spray presence}

In this section we discuss dependence of the coefficients on the effective surface roughness, which is controlled by the waves and sprays. For the estimation of the wave parameters mean square slope (m.s.s.) is used (see (21)). Fig. 8 shows the dependency of drag coefficient and the Stanton number on m.s.s. As it was mentioned before sprays play a key role in the transfer processes. Presence of sprays in the air could be controlled by the detecting spikes in the signals of hot film close to the way used in
[36]. Thus the closed symbols on the plot show the data obtained in no-spray conditions, and the open symbols denote the data obtained in the presence of spray.

It can be seen from the figures that in the absence of spray the drag coefficient increase with the steepness of the wave, the heat transfer coefficient is almost independent of m.s.s.. The same figure shows that both exchange coefficient strongly increase in the presence of spray (open symbols).
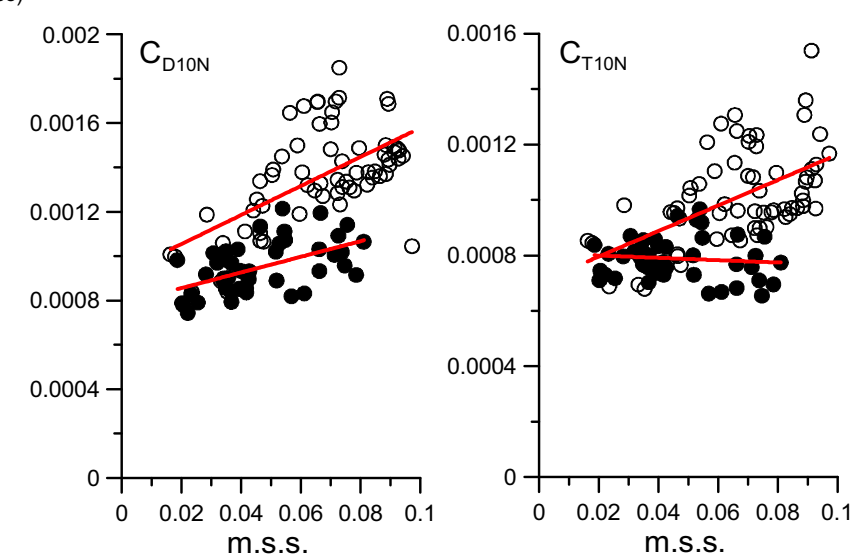

Fig. 8 Dependencies of the drag coefficient (a), heat transfer coefficient (b) on m.s.s Red line - linear approximation. Closed symbols corresponds absence of the sprays in experiments, open symbols corresponds presence of spray.

\section{Conclusion}

The most important characteristics that determine the interaction between the atmosphere and the ocean are flows of momentum, heat and moisture. For their parameterization the dimensionless exchange coefficients (the surface drag coefficient $C_{D 10 N}$ and the heat transfer coefficient or the Stanton number $C_{T 10 N}$ ) are used. Numerous field and laboratory experiments show that $C_{D I 0 N}$ increases with increasing wind speed at moderate and strong wind, and, as was recently shown, $C_{D 10 N}$ decreases at hurricane wind speed. Waves are known to increase the sea surface resistance due to enhanced form drag, the sea spray is considered a possible mechanism of the "drag reduction" at hurricane conditions. The dependence of the heat transfer coefficient $C_{T 10 N}$ on the wind speed is not so certain, and the role of the mechanism associated with the wave disturbances in the mass transfer is not completely understood. The observations and laboratory data show that this dependence is weaker than for the $C_{D 10 N}$, and there are differences in the character of the dependence in different data sets.

A series of experiments to study the processes of turbulent exchange of momentum and heat in a stably stratified temperature turbulent boundary layer air flow over the waved water surface was carried out on the Wind-Wave Flume of the Large Thermostratified Tank of IAP RAS. The peculiarity of this experiment was the option to change the surface wave parameters regardless of the speed of the wind flow in the channel. For this purpose a plastic net has been stretched along the channel. The net does not affect the heat exchange, but 
the characteristics of surface waves depended on the position of the net. To create a stable temperature stratification of the wind, the air entering the flume was heated but the surface water temperature was maintained constant. Experiments were performed for a wide range of wind speed up to hurricane conditions. The turbulent flows of heat and momentum and roughness parameters were retrieved from the measured velocity and temperature profiles and subsequent data processing exploiting the self-similarity of the temperature and velocity profiles. As a result the surface drag and heat exchange coefficients as well as roughness parameters were obtained. Wind wave spectra and integral parameters (significant wave height, mean square slope) were retrieved from measurements by a 3-channel array wave gauge by coherent spectral data processing. The dependences of the exchange coefficients on the winds speed and wave parameters were obtained. It is shown that the exchange coefficients increase with the wind speed and wave height. It was found, that a sharp increase of heat exchange coefficient at wind speeds exceeding $25 \mathrm{~m} / \mathrm{s}$ was accompanied by the emergence of the spray in the air flow over water.

This work was supported by the Russian Foundation of Basic Research (No. 15-35-20953 17-05-00958, 14-0591767 AF_a, 16-55-52022), providing experiments was partial supported by Russian Science Foundation (Agreement No. 14-17-00667) and processing of the data was partially supported by Russian Science Foundation (Agreement No. 15-17-20009).

\section{References}

1. C.W. Fairall, E.F. Bradley, J.E. Hare, A.A. Grachev, J.B. Edson, J. Climate. 16 (2003)

2. Monin, A.S., Yaglom A.M., (Statistical hydrodynamics, NAUKA. 1965).

3. Zilitinkevich, S. S., Izvestiya AN SSSR. FAO, 23, 593 (1976)

4. V.N. Lykossov, Wind stress over the ocean, (Cambridge University Press, 2002)

5. Janssen, P. A. E. M.,J. Phys. Oceanogr., 19, 745 (1989)

6. Makin, V. K., Kudryavtsev, V. N., and Mastenbroek, C., Boundary- Layer Meteorol., 73, 159 (1995)

7. Makin, V. K. and Kudryavtsev, V. N. J. Geophys. Res., 104(C4), 7613(1999)

8. Yu. I. Troitskaya, G. V. Rybushkina, Izvestiya, Atmospheric and Oceanic Physics, 44(5), 621 (2008)

9. Yu.I., Ezhova E.V., Zilitinkevich S.S. Nonlin. Processes Geophys. 20, (2013)

10. Makin V. K. and Mastenbroek C., Boundary-Layer Meteorol., 79, 279 (1996)

11. Makin, V. K., J. Geophys. Res., 103(C1), 1137 (1998)

12. Ocampo-Torres F. J., Donelan M. A., Merzi N., and Jia F, Tellus, Ser. B, 46, 16 (1994).
13. Brut, A., Butet, A., Durand, P., Caniaux, G., and Planton, S., Q.J.R. Meteorol. Soc., 131, 2497 (2005)

14. Dahai Jeong, Brian K. Haus, and Mark A. Donelan,Spray. J. Atmos. Sci., 69, 2733 (2012).

15. Drennan, W. M., Zhang, J., French, J. R., McCormick, C., and Black, P. G., J. Atmos. Sci., 64, 1103 (2007)

16. Andreas E. L., J. Phys. Oceanogr., 28, 2175 (1998)

17. Makin V. K. Boundary Layer Meteorol., 115 (1), 169 (2005)

18. Kudryavtsev V. N. J. Geophys. Res., 111, C07020 (2006)

19. Bortkovskii etl.. (Processi perenosa tepla vblizi poverhnosti ocean-atmosphera, Hydrometizdat, 1974)

20. Kudryavtsev V. N., and V. K. Makin, Boundary Layer Meteorol., 140(3), 383 (2011)

21. Emanuel, K. A., J. Atmos. Sci., 52, 3969 (1995)

22. Yu. I. Troitskaya, D.A. Sergeev, A.A. Kandaurov, G.A Baidakov, M.A. Vdovin, V.I. Kazakov J. Geophys. Res, 117, C00J21 (2012)

23. Yu.I.Troitskaya, D.A.Sergeev, A.A.Kandaurov, M.I. Vdovin, A.A. Kandaurov, E.V.Ezhova, S.S.Zilitinkevich Nonlinear. Geoph. Processes, 20, (2013)

24. Hinze J. O. (Turbulence: An Introduction to its Mechanism and Theory, McGraw-Hill. 1959)

25. Belcher, S.E., Newley, T.M.J. and Hunt J.C.R., J. Fluid Mech., 249, 557 (1993)

26. O. A. Druzhinin Y. I. Troitskaya and S. S. Zilitinkevich Q. J. R. Meteorol. Soc. DOI:10.1002/qj.2677 (2015)

27. Donelan, M.A., W.M. Drennan and A.K. Magnusson, J. Phys. Oceanogr., 26, 1901 (1996)

28. Kudryavtsev V. N., and V. K. Makin BoundaryLayer Meteorology, 98, 155 (2001)

29. DeCosmo, J., K. B. Katsaros, S. D. Smith, R. J. Anderson, W. A. Oost, K. Bumke, and H. Chadwick, J. Geophys. Res., 101, 12,001 (1996)

30. Donelan, M. A., B. K. Haus, N. Reul, W. J. Plant, M. Stiassne, H. C. Graber, O. B. Brown, and E. S. Saltzman (2004), Geophys. Res. Lett., 31, L18306 (2004)

31. Black, P. G. et al., Bull. Am. Meteorol. Soc., 88, 357 (2007)

32. French, J. R., W. M. Drennan, J. A Zhang, and P. G. Black J. Atmos. Sci., 64, 1089 (2007)

33. Powell, M. D., P. J. Vickery, and T. A. Reinhold Nature, 422, 279 (2003)

34. Donelan M.A., Drennan W. M. (Proc. Symp. on Air-Water Gas Transfer, Heidelberg University, 677, 1995)

35. Edson etl Bulletin of the American Meteorological Society. 88 (3), 341 (2007)

36. Lai, R. J., and O. H. Shemdin, J. Geophys. Res., 79, 3055 (1974). 